\title{
Nash implementation with lottery mechanisms
}

\author{
Citation for published version (APA):
}

Bochet, O. L. A. (2005). Nash implementation with lottery mechanisms. METEOR, Maastricht University School of Business and Economics. METEOR Research Memorandum No. 038 https://doi.org/10.26481/umamet.2005038

Document status and date:

Published: 01/01/2005

DOI:

10.26481/umamet.2005038

Document Version:

Publisher's PDF, also known as Version of record

\section{Please check the document version of this publication:}

- A submitted manuscript is the version of the article upon submission and before peer-review. There can be important differences between the submitted version and the official published version of record.

People interested in the research are advised to contact the author for the final version of the publication, or visit the DOI to the publisher's website.

- The final author version and the galley proof are versions of the publication after peer review.

- The final published version features the final layout of the paper including the volume, issue and page numbers.

Link to publication

\footnotetext{
General rights rights.

- You may freely distribute the URL identifying the publication in the public portal. please follow below link for the End User Agreement:

www.umlib.nl/taverne-license

Take down policy

If you believe that this document breaches copyright please contact us at:

repository@maastrichtuniversity.nl

providing details and we will investigate your claim.
}

Copyright and moral rights for the publications made accessible in the public portal are retained by the authors and/or other copyright owners and it is a condition of accessing publications that users recognise and abide by the legal requirements associated with these

- Users may download and print one copy of any publication from the public portal for the purpose of private study or research.

- You may not further distribute the material or use it for any profit-making activity or commercial gain

If the publication is distributed under the terms of Article $25 \mathrm{fa}$ of the Dutch Copyright Act, indicated by the "Taverne" license above, 


\title{
Nash Implementation with Lottery Mechanisms* Olivier Bochet ${ }^{\dagger}$
}

\author{
September 2005
}

\begin{abstract}
Consider the problem of exact Nash Implementation of social choice correspondences. Define a lottery mechanism as a mechanism in which the planner can randomize on alternatives out of equilibrium while pure alternatives are always chosen in equilibrium. When preferences over alternatives are strict, we show that Maskin monotonicity (Maskin, 1999) is both necessary and sufficient for a social choice correspondence to be Nash implementable. We discuss how to relax the assumption of strict preferences. Next, we examine social choice correspondences with private components. Finally, we apply our method to the issue of voluntary implementation (Jackson and Palfrey, 2001).
\end{abstract}

Keywords: Lottery mechanism, Nash implementation, no-veto power, Maskin monotonicity.

\footnotetext{
*I thank Toyo Sakai for his comments on a previous draft of this paper. I also thank two anonymous referees and an editor of this journal for helpful comments that improved this paper. A previous version circulated as "A note on Maskin monotonicity". After the results of this paper were obtained, I became aware of a new unpublished paper by Benoit and Ok (2004). The result of Theorem 2 and the discussion that follows is partially similar to their Theorem 1. Corresponding author: o.bochet@algec.unimaas.nl. Mailing address: Department of Economics. Maastricht University. P.O. box 616. 6200 MD Maastricht, The Netherlands.

${ }^{\dagger}$ Maastricht University and CORE.
} 


\section{Introduction}

The goal of implementation theory is to design institutions that eliminate strategic manipulations, on part of the agents, in order to implement desirable social choice correspondences (henceforth SCCs). Maskin monotonicity (Maskin, 1999) is a necessary condition for (exact) Nash implementation. When there are at least three players, it is also sufficient if coupled with the assumption of no-veto power. ${ }^{1}$ In economic environments with a perfectly divisible good (e.g. money), where there is typically conflict of interest, noveto power is vacuously satisfied since agents will never agree on a best alternative. For more general environments, the gap between necessity and sufficiency was closed by Moore and Repullo (1990), Sjöström (1991) and Danilov (1992), among others. ${ }^{2}$ The necessary and sufficient condition is complex and may be hard to interpret.

We suggest closing the gap between necessity and sufficiency by looking at a different class of mechanisms than the canonical one used in Maskin (1999). We call it the class of lottery mechanisms. While equilibrium messages should still deliver (pure) alternatives selected by the SCC, we allow the planner to use non-degenerate lotteries out of equilibrium. We construct an alternative version of the canonical mechanism in which agents can obtain a lottery on two alternatives for some message profiles. Unlike in Maskin (1999), preferences of agents should also be extended to preference over lotteries. A consequence is that, under some assumptions on the environment, every Maskin monotonic SCC is Nash implementable by a lottery mechanism. The Theorem closes the gap between Maskin monotonicity and Nash implementability. It does not require agents to have preferences satisfying the Von-Neumann and Morgenstern axioms.

In an independent study, Benoit and Ok (2004) address the same question. The mechanism they use belongs to our class of lottery mechanisms. They show that if there are at least three agents and the set of top alternatives is a

\footnotetext{
${ }^{1}$ No-veto power states that if at least $n-1$ agents agree on a best alternative at a preference profile, it should be selected by the SCC at that profile. This condition is restrictive. For example, the individually rational SCC, both in problems of indivisible goods assignment and in voting settings, fail to satisfy it.

${ }^{2}$ Danilov (1992) derives an elegant necessary and sufficient condition for Nash implementation in the case of linear orders on alternatives called essential monotonicity. In such an environment, his condition is equivalent to the condition of Moore and Repullo (1990) or Sjöström (1991).
} 
singleton for at least two agents, then any unanimous and Maskin monotonic SCC is implementable by a lottery mechanism. ${ }^{3}$

In the main theorem, for simplicity, we restrict our attention to linear orderings over (pure) alternatives. Next, we first discuss how we can easily relax the assumption of strict preferences. The domain restriction we introduce is top strict difference. It says that if an alternative is ranked top by at least $(n-1)$ agents, then the set of top alternatives should be a singleton for at least two agents. Moreover, our result does not require that the SCC be unanimous. Second, we examine cases where alternatives are vectors with private components, an issue not covered by Benoit and Ok (2004). ${ }^{4}$ Finally, we consider the case of voluntary implementation (Jackson and Palfrey, 2001) and show that their h-no-veto power axiom can also be dispensed with by considering a lottery mechanism.

\section{The set-up}

There is a fixed finite set of agents $N \equiv\{1, \ldots, n\}$, with $n \geq 3$, and a fixed finite set of alternatives $A$, with $|A| \equiv \ell \geq 2$. Let $\mathcal{L} \equiv \Delta^{\ell-1}$ be the set of lotteries over $A$. In lottery $x=\left(x_{a}\right)_{a \in A} \in \mathcal{L}$, alternative $a$ occurs with probability $x_{a}$. Abusing notation, we write $a$ both for the pure alternative $a \in A$ and for the lottery $x \in \mathcal{L}$ with $x_{a}=1$. The support of a lottery $x \in \mathcal{L}$ is the set of pure alternatives receiving a strictly positive probability in $x: \operatorname{supp} x \equiv\left\{a \in A \mid x_{a}>0\right\}$.

The set of admissible preference profiles over $A$ and over $\mathcal{L}$ are, respectively, $\Theta$ and $\Gamma$. For any $\theta \in \Theta, R_{i}(\theta)$ stands for the (weak) preference of agent $i \in N$ over alternatives in $A$; that is, $R_{i}(\theta)$ is a complete and transitive binary relation. We denote by $P_{i}(\theta)$ and $I_{i}(\theta)$ the associated strict and indifference binary relations, respectively. Similarly, define for any $\gamma \in \Gamma$, $R_{i}(\gamma), P_{i}(\gamma)$ and $I_{i}(\gamma)$ in the same fashion. Given $\theta \in \Theta$, let $\Sigma(\theta) \subseteq \Gamma$ be the set of preferences over $\mathcal{L}$ that agree on the ranking over $A$. We make two assumptions on preferences.

Strictness: For each $\theta \in \Theta$, each $a, b \in A$ and each $i \in N$, if $a R_{i}(\theta) b$, then $a P_{i}(\theta) b$ or $a=b$.

\footnotetext{
${ }^{3}$ They also discuss implementation using mechanisms with awards and implementation with a renegotation function.

${ }^{4}$ E.g., the assignment of indivisible goods without monetary transfers, matching problems, etc...
} 
Monotonicity in probabilities: Preferences over lotteries are monotonic in probabilities, that is, shifts in probability to strictly preferred alternatives yield strictly preferred lotteries. For each $i \in N$, and each $k \in\{1, \ldots, \ell\}$, let $p_{i k}: \Theta \rightarrow A$ be defined by

$$
p_{i k}(\theta)=a \Longleftrightarrow\left|\left\{b \in A \mid b R_{i}(\theta) a\right\}\right|=k .
$$

That is, $p_{i 1}(\theta)$ is the preferred alternative of agent $i$ at profile $\theta, p_{i 2}(\theta)$ her second preferred alternative, etc. Then, if two lotteries $x \equiv\left(x_{a}\right)_{a \in A}$ and $y \equiv\left(y_{a}\right)_{a \in A}$ are such that for each $k^{*} \in\{1, \ldots, \ell\}, \sum_{k \leq k^{*}} x_{p_{i k}(\theta)} \geq \sum_{k \leq k^{*}} y_{p_{i k}(\theta)}$, then $x R_{i}(\gamma) y$ for any $\gamma \in \Sigma(\theta)$, and whenever at least one such inequality is strict, then $x P_{i}(\gamma) y$ for all $\gamma \in \Sigma(\theta)$.

Denote by $L C_{i}(\theta, a)$ the lower contour set of agent $i \in N$ at profile $\theta \in \Theta$ and alternative $a \in A$, i.e.,

$$
L C_{i}(\theta, a) \equiv\left\{b \in A: a R_{i}(\theta) b\right\} .
$$

For each $\theta \in \Theta$, and each $i \in N$, let $T O P_{i}(\theta) \equiv\left\{a \in A: a R_{i}(\theta) b\right.$ for each $\left.b \in A\right\}$.

A social choice correspondence is a mapping $f: \Theta \rightarrow A$ that associates to each preference profile a non-empty subset of alternatives.

Unanimity: A SCC $f$ is unanimous if and only if for each pair $(a, \theta) \in$ $A \times \Theta$,

$$
\left[a R_{i}(\theta) b \text { for each } i \in N \text { and each } b \in A\right] \Longrightarrow[a \in f(\theta)] \text {. }
$$

Notice that unanimity is implied by no-veto power, defined next.

No-Veto power: A SCC $f$ satisfies no-veto power if and only if for each pair $(a, \theta) \in A \times \Theta$, and each $i \in N$,

$$
\left[a R_{j}(\theta) b \text { for each } j \neq i \text { and each } b \in A\right] \Longrightarrow[a \in f(\theta)] \text {. }
$$

Maskin monotonicity: A SCC $f$ is Maskin monotonic (Maskin, 1999) if and only if for each pair $(\theta, \phi) \in \Theta^{2}$ and each $a \in f(\theta)$,

$$
\left[L C_{i}(\theta, a) \subseteq L C_{i}(\phi, a) \text { for each } i \in N\right] \Longrightarrow[a \in f(\phi)] .
$$


For each agent $i \in N$, each $\theta \in \Theta$, and each $a \in A$, define

$$
C_{i}(\theta, a) \equiv\left\{\begin{array}{c}
c \in L C_{i}(\theta, a): \text { for all } \phi \in \Theta, \text { if } L C_{i}(\theta, a) \subseteq L C_{i}(\phi, c) \text { and }, \\
\text { for each } j \neq i, L C_{j}(\phi, c)=A, \text { then } c \in f(\phi) .
\end{array}\right\}
$$

Strong monotonicity: A SCC $f$ is strongly monotonic if and only if for each pair $(\theta, \phi) \in \Theta^{2}$ and $a \in f(\theta)$,

$$
\left[C_{i}(\theta, a) \subseteq L C_{i}(\phi, a) \text { for each } i \in N\right] \Longrightarrow[a \in f(\phi)] .^{5}
$$

A deterministic mechanism (or game form) is a pair $G=(M, g)$ where $M \equiv \underset{i \in N}{\times} M_{i},\left(M_{i}\right.$ is the message space of agent $\left.i \in N\right)$ and $g: M \rightarrow A$ is an outcome function that associates an alternative to every profile of messages.

A mechanism $G$ is a lottery mechanism if for each $\theta \in \Theta$ and each $m \in M$, $g(m) \in \mathcal{L}$. Hence, $g: M \rightarrow \mathcal{L}$. That is, any outcome of the mechanism is a lottery-whether this lottery is degenerate or non-degenerate. Because our focus is on exact implementation of deterministic SCCs, the Nash equilibrium outcomes of the mechanism should be degenerate lotteries. Let $\mathcal{G}$ be the class of lottery mechanisms.

A game for $G$ is a pair $(G, \gamma)$ for some $\gamma \in \Gamma$. We will restrict our attention to pure-strategy Nash equilibria of the game $(G, \gamma)$, denoted $N E(G, \gamma)$. A lottery mechanism $G=(M, g)$ is ordinal if the set of Nash equilibria only depends on agents' preferences over pure alternatives; that is, for each $\theta \in \Theta$, each $m \in M$ and all $\gamma, \delta \in \Sigma(\theta), N E(G, \gamma)=N E(G, \delta)$. We confine our attention to ordinal game forms. Therefore, abusing notation, for any $\gamma \in \Sigma(\theta)$, let $N E(G, \theta)$ denote the pure-strategy Nash equilibria of the game $(G, \gamma)$.

A SCC $f$ is Nash implementable via a lottery mechanism if there exists a mechanism $G \in \mathcal{G}$ such that the Nash equilibrium outcomes of each game coincides with the outcomes chosen by $f$. That is, for each $\theta \in \Theta$ and each $\gamma \in \Sigma(\theta), f(\theta)=g(N E(G, \theta))$.

\section{Enlarging the Class of Mechanisms}

To understand why enlarging the class of admissible mechanisms could help dispensing with the no-veto power assumption, it is useful to recall Maskin's

\footnotetext{
${ }^{5}$ When strictness is satisfied, the essential monotonicity condition of Danilov (1992) coincides with strong monotonicity.
} 
Theorem 3 (Maskin, 1999) and his canonical mechanism. The necessity of Maskin monotonicity for Nash implementation with deterministic mechanisms is omitted from the Theorem statement because the sufficiency part is the focus of our paper.

Theorem 1 (Maskin, 1999) If $n \geq 3$, any Maskin monotonic SCC that satisfies no-veto power is Nash implementable via a deterministic mechanism.

Proof: For each $i \in N$, let $M_{i} \equiv A \times \Theta \times \mathbb{N}$. A typical message is $m_{i} \equiv\left(m_{i}^{1}, m_{i}^{2}, m_{i}^{3}\right) \equiv(a, \theta, n)$. The outcome function is described as follows.

Rule 1: If $m_{i}=(a, \theta, \cdot)$ for all $i \in N$ and $a \in f(\theta)$, then $g(m)=a$.

Rule 2: If for some $i \in N, m_{j}=(a, \theta, \cdot)$ for each $j \neq i, a \in f(\theta)$ and $m_{i}=(b, \phi, \cdot)$ with $(b, \phi) \neq(a, \theta)$, then the outcome is

$$
g(m)=\left\{\begin{array}{l}
b \text { if } b \in L C_{i}(\theta, a) \\
a \text { otherwise }
\end{array}\right.
$$

Rule 3: In all other cases, the outcome is $m_{i^{*}}^{1}$, where $i^{*} \equiv \min \left\{i \in N: n_{i} \geq n_{j} \forall j \in N\right\}$.

The proof of Theorem 1 is well-known, but we sketch it here for the sake of completeness.

Suppose that the true state is $\theta \in \Theta$. We have different cases to consider.

1) $g(m)$ is given by Rule 3 .

Note that any agent $j \neq i^{*}$ could obtain his top-ranked outcome by announcing $n_{j}>n_{i^{*}}$. If $g(m)$ is a Nash equilibrium outcome of the mechanism, then $L C_{i}(\theta, g(m))=A$ for each $i \in N$. By no-veto power, $g(m) \in f(\theta)$.

2) $g(m)$ is given by Rule 2 .

Note that any agent $j \neq i$ could deviate, trigger the integer game and obtain his top-ranked outcome, say $a$, by announcing $n_{j}>n_{k}$ for all $k \neq j$. If $g(m)$ is a Nash equilibrium outcome, then $L C_{j}(\theta, g(m))=A$ for each $j \neq i$. By no-veto power, $g(m) \in f(\theta)$.

3) $g(m)$ is given by Rule 1 .

If $\theta$ is announced truthfully, then $g(m) \in f(\theta)$. So, suppose instead that $m_{i}=(a, \phi, \cdot)$ and $a \in f(\phi)$. Any agent $i$ could deviate and obtain any alternative $b$ such that $b \in L C_{i}(\phi, a)$. If $g(m)$ is a Nash equilibrium outcome, then for each $i \in N, a R_{i}(\phi) b \Longrightarrow a R_{i}(\theta) b$. By Maskin monotonicity, $a \in f(\theta)$. 
To conclude the proof, suppose the true state is $\theta \in \Theta$ and fix $a \in$ $f(\theta)$. We show that each agent $i \in N$ reporting $m_{i}=(a, \theta, 0)$ form a Nash equilibrium of the mechanism with respect to $\theta$. Given $m=\left(m_{i}\right)_{i \in N}$, Rule 1 applies and the outcome is $a \in f(\theta)$. Any agent $j \in N$ can trigger rule 2 by announcing $m_{j}=(b, \phi, \cdot)$ and obtain $b \in L C_{j}(\theta, a)$. By the definition of the lower contour set, such a deviation cannot be profitable. Therefore, $m$ is a Nash equilibrium.

$$
\text { Q.E.D. }
$$

Note that in the proof, no-veto power is in fact used only to rule out undesirable equilibria in Rule 2. In Rule 3, only unanimity-obviously implied by no-veto power-is needed. What happens if no-veto power is not satisfied? ${ }^{6}$ Suppose that the true state is $\theta$ and that messages reported are for each $j \neq i, m_{j}=(a, \phi, \cdot)$ with $a \in f(\phi)$, and $m_{i}=(c, \theta, \cdot)$ with $c \in L C_{i}(\phi, a)$. The outcome is $g(m)=c$ given by Rule 2. If no-veto power is not satisfied and $(c, \theta) \neq(a, \phi)$, it could be the case that for each $j \neq i, L C_{j}(\theta, c)=A$, $L C_{i}(\phi, a) \subseteq L C_{i}(\theta, c)$ and $c \notin f(\theta)$. In such a case, no profitable deviations from $m$ are possible: any agent $j \neq i$ could trigger the integer game but $c$ is top-ranked for any such agent. Furtermore, by changing his message, agent $i$ can only obtain an outcome in $L C_{i}(\phi, a)$, which can be no better than $c$ according to $R_{i}(\theta)$. Thus, message $m$ is a Nash equilibrium. Using the terminology of Maskin and Sjöström (2002), $c$ is an awkward outcome for agent $i$ in $L C_{i}(\phi, a)$. In the canonical mechanism, given message $m \in M$ with $m_{i}=(a, \theta, \cdot)$ for all $i \in N$ and $a \in f(\theta)$, the attainable set from Rule 1 is $L C_{i}(\theta, a)$ for each $i \in N$. When $f$ violates no-veto power, the planner should restrict the attainable sets by removing awkward alternatives. For all $i \in N$, the (personalized) attainable sets $C_{i}(\theta, a)$, as defined in section 3, should be constructed. In Rule 2, for each $\theta \in \Theta$ and each $a \in f(\theta)$, one should replace $L C_{i}(\theta, a)$ by $C_{i}(\theta, a)$ for each $i \in N$. In the environment considered by Maskin, a necessary and sufficient condition for Nash implementation via a deterministic mechanism is strong monotonicity. ${ }^{7}$ Indeed, strong monotonicity implies Maskin monotonicity but the converse is not true. However, Maskin monotonicity together with no-veto power imply strong monotonicity.

\footnotetext{
${ }^{6}$ A similar discussion can be found in the excellent survey of Maskin and Sjöström (2002, Section 3.3). We follow here their terminology.

${ }^{7}$ See Maskin and Sjöström (2002) for a more detailed discussion of strong monotonicity.
} 
By considering a larger class of mechanisms that we call lottery mechanisms, each agent $i$ 's attainable set needs only be $L C_{i}(\theta, a)$. The planner does not need to construct personalized attainable sets by removing every awkward outcome.

To illustrate our approach, we consider an important example from Maskin (1985, 1999) of a SCC that is Maskin monotonic, but does not satisfy no-veto power. We use it here to show that this particular SCC is implementable in Nash equilibrium if the planner uses a lottery mechanism. Thus, checking whether the SCC satisfies Maskin monotonicity is enough to know if it is Nash implementable.

Example 1 (Maskin, 1985 and 1999):

$N \equiv\{1,2,3\}, \Theta \equiv\{\theta, \phi\}$ and $A \equiv\{a, b, c\}$. The preferences are described below.

\begin{tabular}{ccccccc} 
& $\theta$ & & \multicolumn{3}{c}{$\phi$} \\
1 & 2 & 3 & 1 & 2 & 3 \\
$b$ & $a$ & $a$ & $b$ & $c$ & $c$ \\
$a$ & $c$ & $c$ & $c$ & $a$ & $a$ \\
$c$ & $b$ & $b$ & & $a$ & $b$ & $b$
\end{tabular}

The SCC is described as follows. Given $\theta^{\prime} \in \Theta,(i)$ if a majority prefers $a$ to $b$ then $a \in f\left(\theta^{\prime}\right) ;(i i)$ if a majority prefers $b$ to $a$, then $b \in f\left(\theta^{\prime}\right)$; and $(i i i)$ $c \in f\left(\theta^{\prime}\right)$ if $L C_{i}\left(\theta^{\prime}, c\right)=A$ for each $i \in N$. This SCC is Maskin monotonic but does not satisfy no-veto power. It is thus not Nash implementable by Maskin's mechanism. In this example, $f(\theta)=\{a\}=f(\phi)$. Observe that $L C_{1}(\theta, a)=L C_{1}(\phi, c)=\{a, c\}$ and $L C_{i}(\phi, c)=A$ for each $i \in N \backslash\{1\}$. Hence, alternative $c$ is an awkward outcome in $L C_{1}(\theta, a)$. In Maskin's mechanism, if the true state is $\phi$, then the profile of messages $m \in M$, with $m_{j}=(a, \theta, \cdot)$ for $j \neq 1$ and $m_{1}=(c, \phi, \cdot)$ is a Nash equilibrium. Rule 2 prescribes the outcome $c \notin f(\phi)$.

Clearly, what needs to be modified is Rule 2. Instead of the outcome being $c$, suppose Rule 2 gives a lottery $(1-\varepsilon) a+\varepsilon c$, with $\varepsilon \in(0,1)$. By monotonicity in probabilities, when the true state is $\phi$, agents 2 and 3 would rather get $c$ with probability one than a lottery on $a$ and $c$. Therefore, when the true state is $\phi$, the profile $m \in M$, with $m_{j}=(a, \theta, \cdot)$ for $j \neq 1$ and $m_{1}=(c, \phi, \cdot)$ is no longer a Nash equilibrium.

We have just informally shown that we can, on the one hand, dispense with no-veto power, and on the other hand, that we do not need to construct 
restricted attainable sets. In fact, with linear orderings, we only need to check whether a SCC is Maskin monotonic to know if it is Nash implementable. Having possibly non-degenerate lotteries in Rule 2 eliminates the need for no-veto power. Moreover, in the necessary and sufficient condition for Nash implementation, only Maskin monotonicity will have bite. ${ }^{8}$

Before stating the main result, it may be worth mentioning that the next theorem can be understood using the necessary and sufficient conditions for Nash implementation applied to the feasible set $\mathcal{L}$. Notice that no completely mixed lottery in the probability simplex can be awkward because $(n-1)$ players cannot have a top alternative there. The only difference between the attainable sets being $L C_{i}$ or $C_{i}$ is only on the boundary of the simplex. But then continuity of preferences implies the equivalence between strong monotonicity and Maskin monotonicity. We are now ready to state our result.

Theorem 2 If $n \geq 3$ and preferences satisfy strictness and monotonicity in probabilities, any SCC is implementable in Nash equilibrium by a lottery mechanism if and only if it is Maskin monotonic. ${ }^{9}$

Proof: We first prove that if $f$ is implementable by a lottery mechanism, then $f$ is Maskin monotonic. If $f$ is implementable in Nash equilibrium by a lottery mechanism, there exists a mechanism $(M, g)$ that implements it. Consider $\theta \in \Theta$ and $a \in f(\theta)$. Since $f$ is implemented, there is $m \in N E(G, \theta)$ such that $g(m)=a$. Hence, for each $i \in N, a R_{i}(\gamma) g\left(m_{i}^{\prime}, m_{-i}\right)$ for all $m_{i}^{\prime} \in M_{i}$ and for all $\gamma \in \Sigma(\theta)$. Because the equilibrium condition should hold for all $\gamma \in \Sigma(\theta)$, we obtain that for each $i \in N$, for each $m_{i}^{\prime} \in M_{i}, a R_{i}(\theta) b$ for all $b \in \operatorname{supp} g\left(m_{i}^{\prime}, m_{-i}\right)$. Suppose there exists $\phi \in \Theta$ with $a \notin f(\phi)$. Implementation of $f$ requires $m \notin N E(G, \phi)$. Hence, there is $i \in N$ and $m_{i}^{\prime} \in M_{i}$ such that $g\left(m_{i}^{\prime}, m_{-i}\right) P_{i}(\gamma) a$ for some $\gamma \in \Sigma(\phi)$. By monotonicity in probabilities, there exists at least one $b \in \operatorname{supp} g\left(m_{i}^{\prime}, m_{-i}\right)$ such that $b P_{i}(\phi) a$. Since $m$ is a Nash equilibrium under $\theta$ for all $\gamma \in \Sigma(\theta)$, it is the case that $a R_{i}(\theta) b$. Therefore, $f$ is Maskin monotonic.

\footnotetext{
${ }^{8}$ It is clear from the proof of Theorem 1 that unanimity is needed in Rule 3 of Maskin's mechanism. In Theorem 2, we show that unanimity can also be dropped by modifying Rule 3 of Maskin's mechanism.

${ }^{9}$ Alternatively, we could also drop the restriction to ordinal game forms. By dropping this assumption, one can look at a richer set of SCCs that uses cardinal information; i.e. $f: \Gamma \rightarrow \mathcal{L}$. In that case, any cardinal SCC is implementable in Nash equilibrium by a lottery mechanism if and only if it is Maskin monotonic (in the simplex).
} 
We now prove the sufficiency part. We construct the following (ordinal) lottery mechanism. ${ }^{10}$ The message space of each agent $i \in N$ is $M_{i} \equiv A \times$ $\Theta \times A \times \mathbb{N}$. A typical message is $m_{i} \equiv\left(m_{i}^{1}, m_{i}^{2}, m_{i}^{3}, m_{i}^{4}\right) \equiv\left(x, \theta, a, n_{i}\right)$. Fix a number $\varepsilon \in(0,1)$.

Rule 1: If $m_{i}=(\bar{x}, \bar{\theta}, \bar{a},$.$) for all i \in N$ and $\bar{x} \in f(\bar{\theta})$, then $g(m)=\bar{x}$.

Rule 2: If $m_{j}=(\bar{x}, \bar{\theta}, \bar{a}, \cdot)$ for each $j \neq i, x \in f(\bar{\theta})$ and $m_{i}=(c, \phi, b, \cdot)$ with $(c, \phi, b) \neq(\bar{x}, \bar{\theta}, \bar{a})$, then the outcome is

$$
g(m)=\left\{\begin{array}{l}
(1-\epsilon) \bar{x}+\epsilon b \text { if } b \in L C_{i}(\bar{\theta}, \bar{x}) \\
\bar{x} \text { otherwise }
\end{array}\right.
$$

Rule 3: In all other cases, the outcome is $\left(1-\frac{1}{1+n_{i^{*}}}\right) a+\frac{1}{1+n_{i^{*}}} b$ if $m_{i^{*}}=$ $\left(a, \cdot, b, n_{i^{*}}\right)$, where $i^{*}=\min \left\{i \in N: n^{i} \geq n^{j} \forall j \in N\right\}$, and $a \neq b$. Otherwise if $a=b$, the outcome is the lottery that assigns equal weights on all the alternatives in $A$.

We show that this game form Nash implements any SCC $f$ that is Maskin monotonic.

First, suppose that the true profile is $\theta$ and that $x \in f(\theta)$. The message profile $m \in M$ with $m_{i}=(x, \theta, x, 0)$ for each $i \in N$ is a Nash Equilibrium of $G$. By unilaterally deviating, an agent $i \in N$ can only trigger Rule 2 and obtain either $x$ with probability 1 or a lottery on $x$ and another alternative $b \in$ $L C_{i}(\theta, x)$. In that case, the deviation decreases the probability of $x \in f(\theta)$ and increases the probability of a worse alternative $b \in A$. By monotonicity in probabilities, this deviation is not profitable. Therefore, $m$ is a Nash equilibrium.

Second, suppose the true state is $\theta \in \Theta$. We now show that for each $\gamma \in \Sigma(\theta), g(N E(G, \theta)) \subseteq f(\theta)$. We have three cases to consider.

1) $g(m)$ is given by Rule 3 .

We show that there are no equilibrium. If $a \neq b$, the outcome is $g(m)=$ $\left(1-\frac{1}{1+n_{i^{*}}}\right) a+\frac{1}{1+n_{i^{*}}} b$. By strictness and monotonicity in probabilities, even

\footnotetext{
${ }^{10}$ The mechanism uses an integer game. This has been the object of many criticismssee for instance Jackson (1992). The device in Rule 3 works because the set of profitable deviations is open. If such constructions are to be avoided, then the theorem may hold only for unanimous SCCs. Moreover, Jackson (1992) shows that restricting the class of mechanisms may have a severe impact on the class of implementable SCCs. There is no presumption that the sufficiency result here or in Maskin (1999) can be replicated using "well-behaved" mechanisms.
} 
if agents agree on the ranking of $a$ and $b$-with $a$ being top-ranked-any agent $i \in N$ could announce $m_{i}^{\prime}=\left(a, \cdot, b, n_{i}^{\prime}\right)$ with $n_{i}^{\prime}>n_{i^{*}}$ in order to give more weight to $a$ and relatively less weight to $b$. Next, if the outcome $g(m)$ is the uniform distribution over alternatives, again by strictness and monotonicity in probabilities, any agent $i \in N$ would deviate to $m_{i}^{\prime}=\left(a, \cdot, b, n_{i}^{\prime}\right)$, with $a \neq b, n_{i}^{\prime}>n_{i^{*}}, a P_{i}(\theta) b$ and $b P_{i}(\theta) c$ for each $c \neq a$, in order to obtain a lottery restricted to two alternatives.

2) $g(m)$ is given by Rule 2

If $g(m)$ is obtained by Rule 2 , note that any agent $j \neq i$ could deviate by announcing $m_{j}^{\prime}$ with $\left(m_{j}^{1 \prime}, m_{j}^{2 \prime}, m_{j}^{3 \prime}\right) \neq(\bar{x}, \bar{\theta}, \bar{a})$ and $n_{j}^{\prime}>n_{k}$ for all $k \neq j$, trigger Rule 3 and obtain a lottery on his two top alternatives under $\theta$.

We have two cases to consider. In the first case, $g(m) \in \mathcal{L}$ and $\operatorname{supp}(g(m))=$ $\left\{a \in A: x_{a}>0\right\}$ is not a singleton. Because preferences are strict, for each agent, any non-degenerate lottery is dominated by a lottery on the two top alternatives with sufficiently high weight placed on the top alternative. Under Rule 3, for any $j \neq i$ this is obtained by reporting a sufficiently large $n_{j}^{\prime}$. Hence, $g(m)$ is not a Nash equilibrium.

In the second case, $g(m)=\bar{x}$ is a degenerate lottery. Suppose that $\bar{x}$ is a Nash equilibrium outcome for $\theta$ chosen by Rule 2. Agent $i$ can profitably deviate by reporting $\left(c^{\prime}, \phi^{\prime}, b^{\prime}, \cdot\right) \neq(\bar{x}, \bar{\theta}, \bar{a})$ if $b^{\prime} \in L C_{i}(\bar{\theta}, \bar{x})$ and $\left((1-\epsilon) \bar{x}+\epsilon b^{\prime}\right) P_{i}(\gamma) \bar{x}$ for $\gamma \in \Sigma(\theta)$. Because preferences over lotteries are monotone in probabilities, such a profitable deviation does not exist if $\bar{x} R_{i}(\theta) b^{\prime}$ for all $b^{\prime} \in L C_{i}(\bar{\theta}, \bar{x})$. Thus, $L C_{i}(\bar{\theta}, \bar{x}) \subseteq L C_{i}(\theta, \bar{x})$. Moreover, if no agent $j \neq i$ has a profitable deviation, then $L C_{j}(\theta, \bar{x})=A$. Thus, $L C_{j}(\bar{\theta}, \bar{x}) \subseteq$ $L C_{j}(\theta, \bar{x})$ for all $j \neq i$. By Maskin monotonicity, $\bar{x} \in f(\theta)$.

3) $g(m)$ is given by Rule 1 .

If $\theta$ is announced truthfully, then the outcome lies in $f(\theta)$ by Rule 1 . Suppose instead that each $i \in N$ announces $m_{i}=(x, \phi, a, \cdot)$ with $\phi \neq \theta$ and $x \in f(\phi)$. To complete the proof, it is sufficient to show that $L C_{i}(\phi, x) \subseteq$ $L C_{i}(\theta, x)$ for all $i \in N$ because it would then follow from Maskin monotonicity that $x \in f(\theta)$.

On the contrary, suppose that there exists an $i \in N$ and $b \in L C_{i}(\phi, x)$ such that $b P_{i}(\theta) x$. By deviating, agent $i$ can obtain $(1-\epsilon) x+\epsilon b$. In order for this deviation not to be profitable, it must be the case that $x R_{i}(\gamma)((1-\epsilon) x+$ $\epsilon b)$ for all $\gamma \in \Sigma(\theta)$. By monotonicity in probabilities, this contradicts the assumption that $b P_{i}(\theta) x$. 
Q.E.D.

Remark. Given the results on virtual implementation in Abreu and Sen (1990) or Abreu and Matsushima (1992), one could hope that the use of lotteries would relax the necessary condition for Nash implementation. Theorem 2 shows that enlarging the range of the mechanism from $A$ to $\mathcal{L}$ closes the gap between Maskin monotonicity and Nash implementability, provided that preferences satisfy strictness and monotonicity in probabilities. Since the focus is not on approximate but on exact Nash implementation, Maskin monotonicity remains necessary.

Abreu and Matsushima (1994) show that any stochastic social choice function can be exactly implemented in iteratively weakly undominated strategies. However, the existence of small fines that can be levied on players and their assumption that agents have a finite set of preferences satisfying the Von-Neumann Morgenstern axioms are crucial to their result.

\section{Discussion}

\subsection{Relaxing strictness of preferences}

If strictness is not satisfied, the mechanism used to establish Theorem 2 may generate bad equilibria. For instance, suppose that in Rule 3, the outcome is $\left(1-\frac{1}{1+n_{i^{*}}}\right) a+\frac{1}{1+n_{i^{*}}} b$ and alternatives $a$ and $b$ are both top-ranked for every agent. Since $f$ is deterministic, $\left(1-\frac{1}{n_{i^{*}}}\right) a+\frac{1}{n_{i^{*}}} b$ would be an undesirable equilibrium outcome. The same thing happens if the outcome is $(1-\epsilon) \bar{x}+\epsilon b$ determined by Rule 2 .

In order to circumvent this problem, we need an additional restriction on the environment. One such restriction is the following.

Top strict difference: For each $\theta \in \Theta$, and for each $a \in A$ such that $L C_{i}(\theta, a)=A$ for at least $(n-1)$ agents $i \in N$, there exist $j, k \in N$ for whom $T O P_{j}(\theta)=T O P_{k}(\theta)=\{a\}$.

This rules out the undesirable equilibrium outcomes in Rule 2 and Rule 3 of the mechanism used in Theorem 2. To see this, suppose that $\theta \in \Theta$ is the true state, and that $(1-\epsilon) x+\epsilon b$ is the outcome of the game following the report of the message profile $m \in M$. If $L C_{j}(\theta, b)=A$ for each $j$ but possibly $i \in N$, top strict difference guarantees that at least one such $j$ is such that 
$\operatorname{TOP}_{j}(\theta)=\{b\}$, and therefore guarantees that a profitable deviation exists: agent $j$ can trigger the integer game by announcing the highest integer and obtain a preferred lottery. Moreover, if an outcome is determined by Rule 3, again top strict difference rules it out as an equilibrium outcome: there, it is enough to have one agent for whom the set of top alternatives is a singleton.

\subsection{SCCs with private components}

SCCs with private components violating no-veto power include-among othersthe core correspondence, the individually rational correspondence (henceforth the IR correspondence) in the assignment of indivisible goods and the stable rule in matching problems. For all these rules, Theorem 1 does not allow one to check whether or not they are Nash implementable.

The message of Theorem 2 and the discussion that followed is that, given some mild domain restrictions, any Maskin monotonic SCC is Nash implementable by a lottery mechanism. When strictness is satisfied, this result extends to environments where alternatives are vectors with private components. ${ }^{11}$ However, when indifferences are introduced, it is important to note that a lottery mechanism in the way we have defined it in Theorem 2 will not work. First, top strict difference needs to be redefined. From now on, for each $a \in A$, let $a=\left(a_{1}, \ldots, a_{n}\right)$.

Top strict difference for private components: For each $\theta \in \Theta$, and each $a \in A$ such that $a_{i} \in T O P_{i}(\theta)$ for at least $(n-1)$ agents, there exists $j, k \in N$ for whom $\operatorname{TOP}_{j}(\theta)=\left\{a^{\prime}: a_{j}^{\prime}=a_{j}\right\}$ and $\operatorname{TOP}_{k}(\theta)=\left\{a^{\prime}: a_{k}^{\prime}=a_{k}\right\}$.

If top strict difference for private components is satisfied, when there are private components, Maskin monotonic SCCs are not implementable by the lottery mechanism we have constructed in Theorem 2. To see this, let us look at an example for the assignment of indivisible goods without monetary transfers.

Example 2: Assignment of indivisible objects and the individually rational rule.

$N \equiv\{1,2,3,4\}$. There are four objects $a_{1}, a_{2}, a_{3}$ and $a_{4}$. The initial endowment $\omega_{i}$ of each agent $i \in N$ is the object $a_{i}$. An allocation is an

\footnotetext{
${ }^{11}$ Notice that in Rule 2 the requirement $b \in L C_{i}(\theta, a)$ should be read as $b_{i} \in L C_{i}\left(\theta, a_{i}\right)$. In Rule 3 , the requirement $a \neq b$ should be read $a_{i^{*}} \neq b_{i^{*}}$.
} 
assignment of indivisible objects, one to each agent. Formally, an assignment is a bijection $\sigma: N \rightarrow A$. Let $Z$ be the set of assignments. The IR rule is a correspondence such that for each $\theta \in \Theta, f(\theta)=\left\{\sigma \in Z: \sigma_{i} R_{i}(\theta) \omega_{i}\right.$ for each $i \in N\}$. Consider the following preferences over objects.

$\theta$

$\begin{array}{cccccccc}1 & 2 & 3 & 4 & 1 & 2 & 3 & 4 \\ a_{1} & a_{2} & a_{3} a_{4} & a_{3} a_{4} & a_{1} & a_{2} & a_{1} & a_{3} \\ a_{2} & a_{1} & a_{1} & a_{2} & a_{2} & a_{1} & a_{3} a_{2} & a_{4} \\ a_{3} & a_{3} & a_{2} & a_{1} & a_{4} & a_{3} & a_{4} & a_{1} \\ a_{4} & a_{4} & & & a_{3} & a_{4} & & a_{3}\end{array}$

The selection operated by $f$ is $f(\theta)=\left\{\left(a_{1}, a_{2}, a_{3}, a_{4}\right),\left(a_{1}, a_{2}, a_{4}, a_{3}\right)\right\}$, and $f(\phi)=\left\{\left(a_{1}, a_{2}, a_{3}, a_{4}\right)\right\}$. Notice that $f$ violates no-veto power because the allocation $\left(a_{1}, a_{2}, a_{4}, a_{3}\right)$ is excluded when the state is $\phi$. Moreover, $f$ is not Nash implementable by a version of the mechanism in Theorem 2 even though top strict difference for private components is satisfied. Let $a=\left(a_{1}, a_{2}, a_{3}, a_{4}\right)$ and $a^{\prime}=\left(a_{1}, a_{2}, a_{4}, a_{3}\right)$. Consider the case where the true state is $\theta$ and the message $m$ with $m_{j}=\left(a, \phi, a^{\prime}, \cdot\right)$ for $j=2,3,4$ and $m_{1}=\left(\cdot, \theta, a^{\prime}, \cdot\right)$ has been reported. The outcome is $g(m)=(1-\epsilon) a+\epsilon a^{\prime}$, which is a Nash equilibrium outcome under $\theta$, in contradiction with the Nash implementation of $f$. The problem does not come from the violation of noveto power but merely from the fact that top strict difference is vacuous. Agents 1 and 2 meet the restriction imposed by top strict difference for private components but they receive the same object in both allocations. Observe that it is crucial that the number of agents be at least four. With three agents, this problem is never encountered.

As a consequence, the mechanism has to be further complicated. For instance, in Rule 2, if $g(m) \in \mathcal{L}$, then among the $(n-1)$ agents $j \neq i$, it should be the case that at least one is not indifferent between both components he receives. $^{12}$

${ }^{12}$ Rule 3 has to be modified in a similar fashion. One such modification is the following: Rule 3.1: In all other cases, the outcome is $\left(1-\frac{1}{1+n_{i^{*}}}\right) a+\frac{1}{1+n_{i^{*}}} b$ if $m_{i^{*}}=\left(a, \cdot, b, n_{i^{*}}\right)$, where $i^{*}=\min \left\{i \in N: n^{i} \geq n^{j} \forall j \in N\right\}, a_{i^{*}} \neq b_{i^{*}}$ and $a_{j} \neq b_{j}$ for each $j \neq i^{*}$. Otherwise, the outcome is the lottery that assigns equal weights on all the alternatives in A. 
Rule 2.1: If $m_{j}=(x, \theta, a,$.$) for each j \neq i, x \in f(\theta)$ and $m_{i}=(c, \phi, b, \cdot) \neq$ $m_{j}$, then the outcome is $g(m)=(1-\epsilon) x+\epsilon b$ if,

\{1) $b_{i} \neq x_{i}, b_{i} \in L C_{i}\left(\theta, x_{i}\right)$ and,

$\{2)$ there is $j \neq i$ such that $\left\{x_{j}, b_{j}\right\} \nsubseteq T O P_{j}(\phi)$

Otherwise, $g(m)=x$.

Once specific forms of indifferences are allowed, lottery mechanims become more complex. In Rule 2.1, the attainable set of agent $i$ is restricted because some alternatives may be removed from $L C_{i}\left(\theta, x_{i}\right)$. Because of top strict difference with private components, this restriction imposes no constraints on deviations. ${ }^{13}$

\section{Application: voluntary implementation}

Our approach is not restricted only to Maskin's Theorem. In a recent article, Jackson and Palfrey (2001) consider voluntary implementation. The problem related to the enforceability of the outcome function out of equilibrium is studied. Agents are not forced to accept the outcome of the mechanism, and can veto some subset of the set of alternatives. For instance, a statecontingent participation constraint defines a mapping from outcomes vetoed by agents into individually rational outcomes.

First, we need to introduce some additional definitions. ${ }^{14}$ Let $\mathcal{F}$ be the set of all social choice functions over $A$. A reversion function is a mapping $h: \Theta \rightarrow A$ that indicates what the outcome is in the case of a veto by at least one individual. A reversion function $h$ induces a mapping $H: A \times \Theta \times \mathcal{F}$ by

$$
H(a, \theta, h)=\left\{\begin{array}{l}
a \text { if } a R_{i}(\theta) h(\theta) \text { for each } i \in N \\
h(\theta) \text { otherwise }
\end{array}\right.
$$

Given a game form $(M, g)$, a message profile $m$ is an $h$-Nash equilibrium of $(M, g)$ at $\theta$ if for each agent $i \in N$,

$$
H(g(m), \theta, h) R_{i}(\theta) H\left(g\left(m_{i}^{\prime}, m_{-i}\right), \theta, h\right) \forall m_{i}^{\prime} \in M_{i} .
$$

\footnotetext{
${ }^{13}$ Obviously, allowing indifferences-but requiring that for each $\theta \in \Theta, \operatorname{TOP}(\theta)$ is unique for at least $(n-1)$ agents-excludes the complication underlined in example 2 .

${ }^{14}$ We follow the notation introduced by Jackson and Palfrey (2001).
} 
A SCC $f$ is $h$-Nash implementable if there exists a mechanism $(M, g)$ such that, for all $\theta \in \Theta$ :

(1) For each $a \in f(\theta)$, there exists an $h$-Nash equilibrium, $m \in M$, such that $H(g(m), \theta, h)=a$.

(2) If $m \in M$ is an $h$-Nash equilibrium at $\theta$, then $H(g(m), \theta, h) \in f(\theta)$.

An analog to Maskin monotonicity is derived. A SCC $f$ is reversionmonotonic relative to $h$ if for each $\theta \in \Theta$, and each $a \in f(\theta)$, there exists $z \in A$ such that

1. $H(z, \theta, h)=a$.

2. For all $\phi \in \Theta$ such that $H(z, \phi, h) \notin f(\phi)$, there exists $y \in A$ and

$i \in N$ such that $H(z, \theta, h) R_{i}(\theta) H(y, \theta, h)$ and $H(y, \phi, h) P_{i}(\phi) H(z, \phi, h)$.

Reversion monotonicity is indeed necessary for $h$-Nash implementation. Coupled with $h$-no-veto power, it is also sufficient, provided $n \geq 3$.

A SCC $f$ satisfies $h$-no-veto power if for each $(a, \theta) \in A \times \Theta$, and each $i \in N$,

$\left[H(a, \theta, h) R_{j}(\theta) H(b, \theta, h)\right.$ for each $j \neq i$ and each $\left.b \in A\right] \Longrightarrow[H(a, \theta, h) \in f(\theta)]$.

A SCC $f$ satisfies h-unanimity if for each $(a, \theta) \in A \times \Theta$,

$\left[H(a, \theta, h) R_{i}(\theta) H(b, \theta, h)\right.$ for each $i \in N$ and each $\left.b \in A\right] \Longrightarrow[H(a, \theta, h) \in f(\theta)]$.

Again, $h$-no-veto power is not necessary for $h$-Nash implementation. An interesting feature of the voluntary implementation approach is that it is possible to construct non-Maskin monotonic SCCs that are, given $h$, reversionmonotonic relative to $h$. We construct an example of such a SCC that also violates $h$-no-veto power. It is a variant of an example of Jackson and Palfrey (2001). ${ }^{15}$ The example is as follows:

Example 3: $N \equiv\{1,2,3\}, A \equiv\{a, b, c, d\}$ and $\Theta \equiv\{\theta, \phi\}$. The statusquo is $c$. The reversion function is constant across states and equal to the status-quo, that is $h(\theta)=c$ for each $\theta \in \Theta$. The function $H$ maps outcomes that are not individually rational to the status-quo. Hence, we want to perform $I R$-Nash implementation. The preferences over alternatives are described as follows.

\footnotetext{
${ }^{15}$ The SCC in their example satisfies $h-N V P$.
} 


\begin{tabular}{llllll} 
& $\theta$ & & \multicolumn{3}{c}{$\phi$} \\
1 & 2 & 3 & 1 & 2 & 3 \\
$a$ & $b$ & $d$ & $d$ & $b$ & $d$ \\
$d$ & $d$ & $a$ & $a$ & $d$ & $a$ \\
$b$ & $a$ & $c$ & $b$ & $a$ & $b$ \\
$c$ & $c$ & $b$ & $c$ & $c$ & $c$
\end{tabular}

Consider the non-Maskin monotonic SCC $f$, with $f(\theta)=\{a, d\}$ and $f(\phi)=\{a\}$. This SCC is reversion-monotonic relative to $h$. We show that agent 2 experiences a preference reversal relative to $h$ when going from $\theta$ to $\phi$. Since alternative $d$ is individually rational for every agent, $H(d, \theta, h)=$ $H(d, \phi, h)=d$. However, for alternative $b$, by $c P_{3}(\theta) b$, we obtain that $H(b, \theta, h)=c$. Moreover, $H(b, \phi, h)=b$. Thus,

$$
\begin{gathered}
H(d, \theta, h) R_{2}(\theta) H(b, \theta, h) \text { and } H(b, \phi, h) P_{2}(\phi) H(d, \phi, h) ; \text { or } \\
d R_{2}(\theta) c \text { and } b P_{2}(\phi) d
\end{gathered}
$$

Finally, it is easy to see that $f$ does not satisfy $h-$ no-veto power. For agent 1 and $3, L C_{1}(\phi, H(d, \phi, h))=L C_{3}(\phi, H(d, \phi, h))=A$ but $H(d, \phi, h)=$ $d \notin f(\phi)$.

We can state the following Theorem.

Theorem 3: If $n \geq 3$ and preferences satisfy strictness and monotonicity in probablilities, any SCC satisfying h-unanimity is h-implementable by a lottery mechanism if and only if it is reversion monotonic relative to $h$.

Proof: The necessity part is omitted and can be adapted from Jackson and Palfrey (2001).

For the sufficiency part, we construct the following mechanism. The message space of each agent $i \in N$ is $M_{i} \equiv A \times \Theta \times A \times \mathbb{N}$. A typical message will be denoted $m_{i} \equiv\left(m_{i}^{1}, m_{i}^{2}, m_{i}^{3}, m_{i}^{4}\right) \equiv\left(x, \theta, a, n_{i}\right)$. Fix a number $\varepsilon \in(0,1)$. The outcome function is described as follows:

Rule 1: If $m_{i}=(\bar{x}, \bar{\theta}, \bar{a},$.$) for all i \in N, H(\bar{x}, \bar{\theta}, h) \in f(\bar{\theta})$ and $\bar{x}$ satisfies 2 ) in the definition of reversion monotonicity, then $g(m)=\bar{x}$. 
Rule 2: If $m_{j}=(\bar{x}, \bar{\theta}, \bar{a},$.$) for each j \neq i, H(\bar{x}, \bar{\theta}, h) \in f(\bar{\theta}), \bar{x}$ satisfies 2) in the definition of reversion monotonicity and $m_{i}=(c, \phi, b, \cdot)$ with $(c, \phi, b) \neq$ $(\bar{x}, \bar{\theta}, \bar{a})$, then the outcome is

$$
g(m)=\left\{\begin{array}{l}
(1-\epsilon) \bar{x}+\epsilon b \text { if } H(b, \bar{\theta}, h) \in L C_{i}(\bar{\theta}, H(\bar{x}, \bar{\theta}, h)) \\
\bar{x} \text { otherwise }
\end{array}\right.
$$

Rule 3: In all other cases, the outcome is a determined by $m_{i^{*}}=\left(a, \cdot, \cdot, n_{i^{*}}\right)$, where $i^{*}=\min \left\{i \in N: n^{i} \geq n^{j} \forall j \in N\right\}$.

The proof is similar to the proof of Theorem 2 and is thus omitted.

Q.E.D.

The assumption of strict preferences can be relaxed, as before. If the SCC is on alternatives with private components, the discussion in Section 4.2 applies.

\section{Concluding remarks}

1) Extending the class of admissible mechanisms is useful for (exact) Nash implementation. Theorem 2 closes the gap between Maskin monotonicity and Nash implementability, provided that preferences are strict and satisfy monotonicity in probabilities. Moreover, no restriction on the attainable sets has to be constructed.

2) Theorem 2 extends to environments where strictness is relaxed to top strict difference. Neverthless, for environments with private consumption sets or in matching problems, top strict difference for private components is vacuous. In such a case, restricted attainable sets have to be constructed for each agent.

3) Lottery mechanisms can also be useful for alternative implementation approaches. Vartiainen (2003) shows that if preferences are strict, one can randomize out of equilibrium and drop the assumption of no-veto power from the Theorem of Abreu and Sen (1990). ${ }^{16}$ We conjecture that similar results could be obtained in incomplete information settings, for instance with Bayesian implementation. ${ }^{17}$

\footnotetext{
${ }^{16}$ I thank an anonymous referee for pointing out this paper.

${ }^{17}$ See for instance Jackson (1991) for a discussion of Bayesian implementation.
} 
4) A possible extension of this work would be to design simple lottery mechanisms to implement SCCs violating no-veto power.

\section{References}

1. Abreu D., Matsushima H., 1992. "Virtual Implementation in Iteratively Undominated Strategies: Complete Information." Econometrica, 60, 993-1008.

2. Abreu D., Matsushima H., 1994. "Exact Implementation." Journal of Economic Theory, 64, 1-19.

3. Abreu D., Sen A., 1990. "Subgame Perfect Implementation: a Necessary and Almost Sufficient Condition.". Journal of Economic Theory, 50, 285-299.

4. Abreu D., Sen A., 1991. "Virtual Implementation in Nash Equilibrium." Econometrica, 59, 997-1021.

5. Benoit J.P., Ok E., 2004. "Nash Implementation without No-Veto Power." Mimeo, Department of Economics, New York University.

6. Danilov V., 1992. "Implementation via Nash Equilibria." Econometrica, 60, 43-56.

7. Jackson M. 1991. "Bayesian Implementation" Econometrica, 59, 461477.

8. Jackson M., Palfrey T., 2001. "Voluntary Implementation." Journal of Economic Theory, 98, 1-25.

9. Kara T, Sonmez T., 1996. "Nash Implementation of Matching Rules." Journal of Economic Theory, 68, 425-439.

10. Maskin E., Sjöström T., 2002. "Implementation Theory." in Handbook of Social Choice and Welfare, Vol. 1, K. Arrow, A. Sen, and K. Suzumura (eds.), Amsterdam North-Holland, 237-288.

11. Maskin E., 1999. "Nash Implementation and Welfare Optimality." Review of Economic Studies, 66, 23-38.

12. Maskin E., 1985. "The Theory of Implementation in Nash Equilibrium: a Survey." in Social Goals and Organization, L. Hurwicz, D. Schmeidler and H. Sonnenschein (eds.), Cambridge: University Press, 173-204.

13. Moore J. and Repullo R. "Nash Implementation: a Full Characterization." Econometrica, 58, 1083-1100.

14. Sjöström T., 1991. "On the Necessary and Sufficient Conditions for Nash Implementation." Social Choice and Welfare, 8, 333-340.

15. Vartiainen H., 2003. "Subgame Perfect Implementation: A Full Characterization and Applications to Voting." Manuscript, University of Helsinki. 
Proposition 1: If $n \geq 3$, and preferences satisfy top strict difference and monotonicity in probabilities, any SCC is Nash implementable by a lottery mechanism if and only if it is Maskin monotonic.

Proof: The necessity part is omitted. It already appears in Theorem 2. For the sufficiency part, we use the same mechanism constructed in Theorem 2 .

The message space of each agent $i \in N$ is $M_{i} \equiv A \times \Theta \times A \times \mathbb{N}$. A typical message is $m_{i} \equiv\left(m_{i}^{1}, m_{i}^{2}, m_{i}^{3}, m_{i}^{4}\right) \equiv\left(x, \theta, a, n_{i}\right)$. Fix a number $\varepsilon \in(0,1)$.

Rule 1: If $m_{i}=(\bar{x}, \bar{\theta}, \bar{a},$.$) for all i \in N$ and $\bar{x} \in f(\bar{\theta})$, then $g(m)=\bar{x}$.

Rule 2: If $m_{j}=(\bar{x}, \bar{\theta}, \bar{a}, \cdot)$ for each $j \neq i, \bar{x} \in f(\bar{\theta})$ and $m_{i}=(c, \phi, b, \cdot)$ with $(c, \phi, b) \neq(\bar{x}, \bar{\theta}, \bar{a})$, then the outcome is

$$
g(m)=\left\{\begin{array}{l}
(1-\epsilon) \bar{x}+\epsilon b \text { if } b \in L C_{i}(\bar{\theta}, \bar{x}) \\
\bar{x} \text { otherwise }
\end{array}\right.
$$

Rule 3: In all other cases, the outcome is $\left(1-\frac{1}{1+n_{i^{*}}} a\right)+\frac{1}{1+n_{i^{*}}} b$ if $m_{i^{*}}=$ $\left(a, \cdot, b, n_{i^{*}}\right)$, where $i^{*}=\min \left\{i \in N: n^{i} \geq n^{j} \forall j \in N\right\}$, and $a \neq b$. Otherwise if $a=b$, the outcome is the lottery that assigns equal weights on all the alternatives in $A$.

We show that this game form Nash implements any SCC $f$ that is Maskin monotonic.

First, suppose that the true profile is $\theta$ and that $x \in f(\theta)$. The message profile $m \in M$ with $m_{i}=(x, \theta, x, 0)$ for each $i \in N$ is a Nash Equilibrium of $G$. By unilaterally deviating, an agent $i \in N$ can only trigger Rule 2 and obtain either $x$ with probability 1 or a lottery on $x$ and another alternative $b \in$ $L C_{i}(\theta, x)$. In that case, the deviation decreases the probability of $x \in f(\theta)$ and increases the probability of a worse alternative $b \in A$. By monotonicity in probabilities, this deviation is not profitable. Therefore, $m$ is a Nash equilibrium.

Second, suppose the true state is $\theta \in \Theta$. We now show that for each $\gamma \in \Sigma(\theta), g(N E(G, \theta)) \subseteq f(\theta)$. We have three cases to consider.

1) $g(m)$ is given by Rule 3 .

We show that there are no equilibrium. If $a \neq b$, the outcome is $g(m)=$ $\left(1-\frac{1}{1+n_{i^{*}}}\right) a+\frac{1}{1+n_{i^{*}}} b$. By top strict difference, even if $a \in T O P_{j}(\theta)$ for at least $(n-1)$ agents $j$, there exists an agent $i$ for whom $b \notin T O P_{i}(\theta)$. Such an 
agent $i$ could announce $m_{i}^{\prime}=\left(a, \cdot, b, n_{i}^{\prime}\right)$ with $n_{i}^{\prime}>n_{i^{*}}$ in order to give more weight to $a$ and relatively less weight to $b$. By monotonicity in probabilities, this is a profitable deviation for agent $i$. The case where $b \in T O P_{j}(\theta)$ for at least $(n-1)$ agents $j$ is similar.

Next, if the outcome $g(m)$ is the uniform distribution over alternatives, again by top strict difference and monotonicity in probabilities, some agent $i \in N$ would deviate to $m_{i}^{\prime}=\left(a, \cdot, b, n_{i}^{\prime}\right)$, with $a \neq b, n_{i}^{\prime}>n_{i^{*}}, a R_{i}(\theta) b$ and $b R_{i}(\theta) c$ for each $c \neq a$, in order to obtain a lottery restricted to two alternatives.

2) $g(m)$ is given by Rule 2

If $g(m)$ is obtained by Rule 2 , note that any agent $j \neq i$ could deviate by announcing $m_{j}^{\prime}$ with $\left(m_{j}^{1 \prime}, m_{j}^{2 \prime}, m_{j}^{3 \prime}\right) \neq(\bar{x}, \bar{\theta}, \bar{a})$ and $n_{j}^{\prime}>n_{k}$ for all $k \neq j$, trigger Rule 3 and obtain a lottery on his two top alternatives under $\theta$.

We have two cases to consider. In the first case, $g(m) \in \mathcal{L}$ and $\operatorname{supp}(g(m))=$ $\left\{a \in A: x_{a}>0\right\}$ is not a singleton. That is, $g(m)=(1-\epsilon) \bar{x}+\epsilon b$. Top strict difference guarantees that even if $\{\bar{x}, b\} \subseteq T O P_{k}(\theta)$ for $(n-1)$ agents $k$, there exists $j \neq i$ such that $\{\bar{x}, b\} \nsubseteq T O P_{j}(\theta)$. For such an agent $j \in N$, $g(m)$ is dominated by a lottery on the two top alternatives with sufficiently high weight placed on the top alternative. Under Rule 3 , for any $j \neq i$ this is obtained by reporting a sufficiently large $n_{j}^{\prime}$. Hence, $g(m)$ is not a Nash equilibrium.

In the second case, $g(m)=\bar{x}$ is a degenerate lottery. Suppose that $\bar{x}$ is a Nash equilibrium outcome for $\theta$ chosen by Rule 2. Agent $i$ can profitably deviate by reporting $\left(c^{\prime}, \phi^{\prime}, b^{\prime}, \cdot\right) \neq(\bar{x}, \bar{\theta}, \bar{a})$ with $b^{\prime} \in L C_{i}(\bar{\theta}, \bar{x})$ and $\left((1-\epsilon) \bar{x}+\epsilon b^{\prime}\right) P_{i}(\gamma) \bar{x}$ for $\gamma \in \Sigma(\theta)$. Because preferences over lotteries are monotonic in probabilities, such a profitable deviation does not exist if $\bar{x} R_{i}(\theta) b^{\prime}$ for all $b^{\prime} \in L C_{i}(\bar{\theta}, \bar{x})$. Thus, $L C_{i}(\bar{\theta}, \bar{x}) \subseteq L C_{i}(\theta, \bar{x})$. Moreover, if no agent $j \neq i$ has a profitable deviation, then $L C_{j}(\theta, \bar{x})=A$. Thus, $L C_{j}(\bar{\theta}, \bar{x}) \subseteq L C_{j}(\theta, \bar{x})$ for all $j \neq i$. By Maskin monotonicity, $\bar{x} \in f(\theta)$.

3) $g(m)$ is given by Rule 1 .

If $\theta$ is announced truthfully, then the outcome lies in $f(\theta)$ by Rule 1 . Suppose instead that each $i \in N$ announces $m_{i}=(x, \phi, a, \cdot)$ with $\phi \neq \theta$ and $x \in f(\phi)$. To complete the proof, it is sufficient to show that $L C_{i}(\phi, x) \subseteq$ $L C_{i}(\theta, x)$ for all $i \in N$ because it would then follow from Maskin monotonicity that $x \in f(\theta)$.

On the contrary, suppose that there exists an $i \in N$ and $b \in L C_{i}(\phi, x)$ 
such that $b P_{i}(\theta) x$. By deviating, agent $i$ can obtain $(1-\epsilon) x+\epsilon b$. In order for this deviation not to be profitable, it must be the case that $x R_{i}(\gamma)((1-\epsilon) x+$ $\epsilon b)$ for all $\gamma \in \Sigma(\theta)$. By monotonicity in probabilities, this contradicts the assumption that $b P_{i}(\theta) x$.

Q.E.D. 
Proposition 2: If $n \geq 3$ and preferences satisfy top strict difference for private components and monotonicity in probabilities, any SCC with private components is Nash implementable by a lottery mechanism if and only if it is Maskin monotonic

Proof: The necessity part is omitted. It already appears in Theorem 2. For the sufficiency part, we use the following mechanism.

The message space of each agent $i \in N$ is $M_{i} \equiv A \times \Theta \times A \times \mathbb{N}$. A typical message is $m_{i} \equiv\left(m_{i}^{1}, m_{i}^{2}, m_{i}^{3}, m_{i}^{4}\right) \equiv\left(x, \theta, a, n_{i}\right)$. Fix a number $\varepsilon \in(0,1)$.

Rule 1: If $m_{i}=(\bar{x}, \bar{\theta}, \bar{a},$.$) for all i \in N$ and $\bar{x} \in f(\bar{\theta})$, then $g(m)=\bar{x}$.

Rule 2.1: If $m_{j}=(\bar{x}, \bar{\theta}, \bar{a},$.$) for each j \neq i, \bar{x} \in f(\bar{\theta})$ and $m_{i}=(c, \phi, b, \cdot)$ with $(c, \phi, b) \neq(\bar{x}, \bar{\theta}, \bar{a})$, then the outcome is $g(m)=(1-\epsilon) \bar{x}+\epsilon b$ if,

$\left\{\begin{array}{l}\text { 1) } b_{i} \neq \bar{x}_{i}, b_{i}^{\prime} \in L C_{i}\left(\bar{\theta}, \bar{x}_{i}\right) \text { and } \\ \text { 2) there is } j \neq i \text { such that }\left\{\bar{x}_{j}, b_{j}\right\} \nsubseteq T O P_{j}(\phi)\end{array}\right.$

Otherwise, $g(m)=x$.

Rule 3.1: In all other cases, the outcome is $\left(1-\frac{1}{1+n_{i^{*}}}\right) a+\frac{1}{1+n_{i^{*}}} b$ if $m_{i^{*}}=$ $\left(a, \cdot, b, n_{i^{*}}\right)$, where $i^{*}=\min \left\{i \in N: n^{i} \geq n^{j} \forall j \in N\right\}, a_{i^{*}} \neq b_{i^{*}}$ and $a_{j} \neq b_{j}$ for each $j \neq i^{*}$. Otherwise, the outcome is the lottery that assigns equal weights on all the alternatives in $A$.

We show that this game form Nash implements any SCC with private components $f$ that is Maskin monotonic.

First, suppose that the true profile is $\theta$ and that $x \in f(\theta)$. The message profile $m \in M$ with $m_{i}=(x, \theta, x, 0)$ for each $i \in N$ is a Nash Equilibrium of $G$. By unilaterally deviating, an agent $i \in N$ can only trigger Rule 2 and obtain either $x_{i}$ with probability 1 or a lottery on $x_{i}$ and another alternative $b_{i} \in L C_{i}\left(\theta, x_{i}\right)$. In that case, the deviation decreases the probability of $x \in f(\theta)$ and increases the probability of a worse alternative $b \in A$. By monotonicity in probabilities, this deviation is not profitable. Therefore, $m$ is a Nash equilibrium.

Second, suppose the true state is $\theta \in \Theta$. We now show that for each $\gamma \in \Sigma(\theta), g(N E(G, \theta)) \subseteq f(\theta)$. We have three cases to consider.

1) $g(m)$ is given by Rule 3 .

We show that there are no equilibrium. Suppose first that the outcome is $g(m)=\left(1-\frac{1}{1+n_{i^{*}}}\right) a+\frac{1}{1+n_{i^{*}}} b$. We have three cases to consider. 
a) $\left\{a_{k}\right\} \subseteq T O P_{k}(\theta)$ for $(n-1)$ agents $k$. By top strict difference for private components, there exists an agent $i \in N$ for whom $\operatorname{TOP}_{i}(\theta)=\left\{a_{i}^{\prime}\right.$ : $\left.a_{i}^{\prime}=a_{i}\right\}$

Since $a_{j} \neq b_{j}$ for each $j \in N$,such an agent $i$ could announce $m_{i}^{\prime}=$ $\left(a, \cdot, b, n_{i}^{\prime}\right)$ with $n_{i}^{\prime}>n_{i^{*}}$ in order to give more weight to $a$ and relatively less weight to $b$. By monotonicity in probabilities, this is a profitable deviation for agent $i$.

b) $\left\{b_{k}\right\} \subseteq T O P_{k}(\theta)$ for $(n-1)$ agents $k$. By top strict difference for private components, there exists an agent $i \in N$ for whom $\operatorname{TOP}_{i}(\theta)=\left\{b_{i}^{\prime}\right.$ : $\left.b_{i}^{\prime}=b_{i}\right\}$.

Since $a_{j} \neq b_{j}$ for each $j \in N$, such an agent $i$ could announce $m_{i}^{\prime}=$ $\left(b, \cdot, a, n_{i}^{\prime}\right)$ with $n_{i}^{\prime}>n_{i^{*}}$ in order to give more weight to $b$ and relatively less weight to $a$. By monotonicity in probabilities, this is a profitable deviation for agent $i$.

c) $\left\{a_{i}, b_{i}\right\} \subseteq \operatorname{TOP}_{i}(\theta)$ for some $i \in N$. By top strict difference for private components, there exists an agent $j \neq i$ for whom $\left\{a_{j}, b_{j}\right\} \nsubseteq$ $\operatorname{TOP}_{j}(\theta)$. Such an agent $j$ could announce $m_{j}^{\prime}=\left(c, \cdot, d, n_{i}^{\prime}\right)$ with $n_{j}^{\prime}>n_{i^{*}}$, $c_{j} \neq d_{j}, c_{j} R_{j}(\theta) d_{j}$ and $d_{j} R_{j}(\theta) e_{j}$ for all $e_{j} \neq c_{j}$, and such that $c_{k} \neq d_{k}$ for each $k \neq j$. By monotonicity in probabilities, this is a profitable deviation for agent $i$.

Next, if the outcome $g(m)$ is the uniform distribution over alternatives, again by top strict difference for private components and monotonicity in probabilities, some agent $i \in N$ would deviate to $m_{i}^{\prime}=\left(a, \cdot, b, n_{i}^{\prime}\right)$, with $a_{i} \neq b_{i}, a_{j} \neq b_{j}$ for each $j \neq i, n_{i}^{\prime}>n_{i^{*}}, a_{i} R_{i}(\theta) b_{i}$ and $b_{i} R_{i}(\theta) c_{i}$ for each $c_{i} \neq a_{i}$, in order to obtain a lottery restricted on two alternatives.

2) $g(m)$ is given by Rule 2

If $g(m)$ is obtained by Rule 2 , note that any agent $j \neq i$ could deviate by announcing $m_{j}^{\prime}$ with $\left(m_{j}^{1 \prime}, m_{j}^{2 \prime}, m_{j}^{3 \prime}\right) \neq(\bar{x}, \bar{\theta}, \bar{a})$ and $n_{j}^{\prime}>n_{k}$ for all $k \neq j$, trigger Rule 3 and obtain a lottery on his two top alternatives under $\theta$.

We have two cases to consider. In the first case, $g(m) \in \mathcal{L}$ and $\operatorname{supp}(g(m))=$ $\left\{a \in A: x_{a}>0\right\}$ is not a singleton. That is, $g(m)=(1-\epsilon) \bar{x}+\epsilon b$. Top strict difference for private components guarantees that even if $\left\{\bar{x}_{k}, b_{k}\right\} \subseteq T O P_{k}(\theta)$ for $(n-1)$ agents $k$, there exists $j \neq i$ such that $\left\{\bar{x}_{j}, b_{j}\right\} \nsubseteq T O P_{j}(\theta)$. For such an agent $j, g(m)$ is dominated by a lottery on the two top alternatives with sufficiently high weight placed on the top alternative. Under Rule 3, for 
any $j \neq i$ this is obtained by reporting a sufficiently large $n_{j}^{\prime}$. Hence, $g(m)$ is not a Nash equilibrium.

In the second case, $g(m)=\bar{x}$ is a degenerate lottery. Suppose that $\bar{x}$ is a Nash equilibrium outcome for $\theta$ chosen by Rule 2. Agent $i$ can profitably deviate by reporting $\left(c^{\prime}, \phi^{\prime}, b^{\prime}\right) \neq(\bar{x}, \bar{\theta}, \bar{a})$ if $b_{i}^{\prime} \in L C_{i}\left(\bar{\theta}, \bar{x}_{i}\right)$ and $\left((1-\epsilon) \bar{x}_{i}+\epsilon b_{i}^{\prime}\right) P_{i}(\gamma) \bar{x}_{i}$ for $\gamma \in \Sigma(\theta)$. Because preferences over lotteries are monotone in probabilities, such a profitable deviation does not exist if $\bar{x}_{i} R_{i}(\theta) b_{i}^{\prime}$ for all $b_{i}^{\prime} \in L C_{i}\left(\bar{\theta}, \bar{x}_{i}\right)$. Thus, $L C_{i}\left(\bar{\theta}, \bar{x}_{i}\right) \subseteq L C_{i}\left(\theta, \bar{x}_{i}\right)$. Moreover, if no agent $j \neq i$ has a profitable deviation, then $\bar{x}_{j} \in T O P_{j}(\theta)$. Thus, $L C_{j}\left(\bar{\theta}, \bar{x}_{j}\right) \subseteq L C_{j}\left(\theta, \bar{x}_{j}\right)$ for all $j \neq i$. By Maskin monotonicity, $\bar{x} \in f(\bar{\theta})$.

3) $g(m)$ is given by Rule 1 .

If $\theta$ is announced truthfully, then the outcome lies in $f(\theta)$ by Rule 1 . Suppose instead that each $i \in N$ announces $m_{i}=(x, \phi, a, \cdot)$ with $\phi \neq \theta$ and $x \in f(\phi)$. To complete the proof, it is sufficient to show that $L C_{i}\left(\phi, x_{i}\right) \subseteq$ $L C_{i}\left(\theta, x_{i}\right)$ for all $i \in N$ because it would then follow from Maskin monotonicity that $x \in f(\theta)$.

On the contrary, suppose that there exists an $i \in N$ and $b_{i} \in L C_{i}\left(\phi, x_{i}\right)$ such that $b_{i} P_{i}(\theta) x_{i}$. By deviating, agent $i$ can obtain $(1-\epsilon) x_{i}+\epsilon b_{i}$. In order for this deviation not to be profitable, it must be the case that $x_{i} R_{i}(\gamma)\left((1-\epsilon) x_{i}+\right.$ $\left.\epsilon b_{i}\right)$ for all $\gamma \in \Sigma(\theta)$. By monotonicity in probabilities, this contradicts the assumption that $b_{i} P_{i}(\theta) x_{i}$.

Q.E.D. 
Proposition 3: If $n \geq 3$ and preferences satisfy strictness and monotonicity in probabilities, any SCC satisfying h-unanimity is $h$-implementable by a lottery mechanism if and only if it is reversion monotonic relative to $h$.

Proof: The necessary part is omitted. The message space of each agent $i \in N$ is $M_{i} \equiv A \times \Theta \times A \times \mathbb{N}$. A typical message will be denoted $m_{i} \equiv$ $\left(m_{i}^{1}, m_{i}^{2}, m_{i}^{3}, m_{i}^{4}\right) \equiv\left(x, \theta, a, n_{i}\right)$. Fix a number $\varepsilon \in(0,1)$. The outcome function is described as follows:

Rule 1: If $m_{i}=(\bar{x}, \bar{\theta}, \bar{a},$.$) for all i \in N, H(\bar{x}, \bar{\theta}, h) \in f(\bar{\theta})$ and $\bar{x}$ satisfies $2)$ in the definition of reversion monotonicity, then $g(m)=\bar{x}$.

Rule 2: If $m_{j}=(\bar{x}, \bar{\theta}, \bar{a},$.$) for each j \neq i, H(\bar{x}, \bar{\theta}, h) \in f(\bar{\theta}), \bar{x}$ satisfies $2)$ in the definition of reversion monotonicity and $m_{i}=\left(c, \phi, b, n_{i}\right)$ with $(c, \phi, b) \neq(\bar{x}, \bar{\theta}, \bar{a})$, then the outcome is

$$
g(m)=\left\{\begin{array}{l}
(1-\epsilon) \bar{x}+\epsilon b \text { if } H(b, \bar{\theta}, h) \in L C_{i}(\bar{\theta}, H(\bar{x}, \bar{\theta}, h)) \\
\bar{x} \text { otherwise }
\end{array}\right.
$$

Rule 3: In all other cases, the outcome is a given by $m_{i^{*}}=\left(a, \cdot, \cdot, n_{i^{*}}\right)$, where $i^{*}=\min \left\{i \in N: n^{i} \geq n^{j} \forall j \in N\right\}$.

First, suppose that the true profile is $\theta$ and that, given $h$, there exists $x$ such that $H(x, \theta, h) \in f(\theta)$. The message profile $m \in M$ with $m_{i}=(x, \theta, x, 0)$ for each $i \in N$ is a Nash Equilibrium of $G$. By unilaterally deviating, an agent $i \in N$ can only trigger Rule 2 and obtain either $H(x, \theta, h)$ with probability 1 or a lottery on $H(x, \theta, h)$ and another alternative $H(b, \theta, h) \in L C_{i}(\theta, H(x, \theta, h))$. In that case, the deviation decreases the probability of $H(x, \theta, h) \in f(\theta)$ and increases the probability of a worse alternative $H(b, \theta, h) \in A$. By monotonicity in probabilities, this deviation is not profitable. Therefore, $m$ is a Nash equilibrium.

Second, suppose the true state is $\theta \in \Theta$. We now show that for each $\gamma \in \Sigma(\theta), g(N E(G, \theta)) \subseteq f(\theta)$. We have three cases to consider.

1) $g(m)$ is given by Rule 3

Any agent $i$ could deviate by announcing $n_{i}>n_{i^{*}}$ and cause the outcome function to choose any outcome in $A$. If for all agent $i \in N$ such a deviation does not exist, this implies that for each $i \in N, H(g(m), \theta, h) R_{i}(\theta) H(y, \theta, h)$ for all $y \in A$. By $h$-unanimity of $f$, this implies that $H(g(m), \theta, h) \in f(\theta)$.

2) $g(m)$ is given by Rule 2 
We have two cases to consider. In the first case, $g(m) \in \mathcal{L}, \operatorname{supp}(g(m))$ is not a singleton and $H(\bar{x}, \theta, h) \neq H(b, \theta, h)$. By strictness, any agent $j \neq i$ could deviate by triggering Rule 3 and obtain, with probability one, the alternative he prefers between $H(\bar{x}, \theta, h)$ and $H(\bar{b}, \theta, h)$. Hence, $g(m)$ is not a Nash equilibrium.

In the second case, either $g(m)=\bar{x}$, or $H(\bar{x}, \theta, h)=H(b, \theta, h)$. Suppose that $g(m)$ is a Nash equilibrium outcome under $\theta$. Then, it is the case that $H(\bar{x}, \theta, h) \in f(\theta)$. Otherwise, by the definition of reversionmonotonicity, there would exist an agent $k \in N$ and $y \in A$ such that $H(y, \bar{\theta}, h) \in L C_{k}\left(\bar{\theta}, H(\bar{x}, \bar{\theta}, h)\right.$ and $H(y, \theta, h) P_{k}(\theta) H(\bar{x}, \theta, h)$.

1) $g(m)$ is given by Rule 1

If $\theta$ is announced truthfully, then $H(g(m), \theta, h) \in f(\theta)$ by Rule 1 . So suppose instead that each $i \in N$ announces $m_{i}=(x, \phi, a, \cdot)$ with $\phi \neq \theta$ and $H(x, \phi, h) \in f(\phi)$.

Then, it is the case that $H(x, \theta, h) \in f(\theta)$. Otherwise, by the definition of reversion-monotonicity, there would exist an agent $i \in N$ and $y \in A$ such that $H(y, \phi, h) \in L C_{i}(\phi, H(x, \phi, h))$ and $H(y, \theta, h) P_{i}(\theta) H(x, \theta, h)$.

Q.E.D. 